\title{
Declínio cognitivo de idosos e sua associação com fatores epidemiológicos em Viçosa, Minas Gerais \\ (1)
}

\author{
Cognitive decline of aged and its association with epidemiological \\ factors in the city of Viçosa, Minas Gerais
}

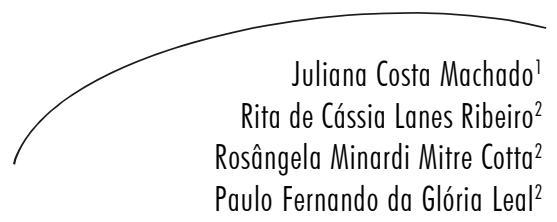

Resumo

Introdução: Atualmente, a demência representa um significativo problema de saúde pública pela longa extensão e complexidade de manifestações funcionais, emocionais e consequências sociais, tanto para os idosos quanto para seus familiares. O diagnóstico de demência tem como base a investigação do declínio cognitivo em populações de risco. Objetivo: Esta pesquisa teve como objetivo avaliar o estado mental de pacientes com 60 anos ou mais, frequentadores do Programa Municipal da Terceira Idade, em Viçosa MG, e sua relação com os fatores relacionados à situação de saúde. Métodos: Realizou-se um estudo transversal com 74 idosos. Foram excluídos os idosos sem escolaridade. O procedimento de coleta de dados adotado foi a aplicação do questionário para a avaliação das características epidemiológicas e dos fatores relacionados à saúde e o teste do Mini-Exame do Estado Mental. Resultados: Dentre os idosos avaliados, encontrou-se uma prevalência de declínio cognitivo de $36,5 \%$. A média de pontuação no MEEM foi de 19,48 pontos e a média da idade dos idosos foi de 68,5 anos. Discussão: Os resultados encontrados mostram que o menor nível de escolaridade tem influência negativa no exame do estado mental dos idosos. Conclusão: Conclui-se que é alto o número de idosos identificados com declínio cognitivo, resultado que revela que o Teste MEEM é influenciado pela variável escolaridade e, como mostrado em outros estudos, o ponto de corte utilizado deve ser ajustado à escolaridade.

\section{Abstract}

Introduction: Currently, dementia is a meanly problem in public health due to the long extension and complexity of functional and emotional manifestations and social consequence, to the elderly and their relatives. The diagnosis of dementia is based on investigation in the cognitive decrease in risk population. Objective;

\footnotetext{
Programa de Pós-graduação em Ciências da Nutrição. Departamento de Nutrição e Saúde, Universidade Federal de Viçosa. Viçosa, MG, Brasil

2 Departamento de Nutrição e Saúde, Universidade Federal de Viçosa, Viçosa, MG, Brasil

Correspondência / Correspondece

Juliana Costa Machado

E-mail: juboechat@yahoo.com.br
}

Palavras-chave: Idoso. Avaliação. Declínio Cognitivo. Mini-Exame do Estado Mental. Demência. 
This research aims to evaluate the mental state in patients aged 60 years old or more, participants in the City Program for the Elderly, in Viçosa MG, and their relation with the factors concerning the health situation. Methods: A cross-sectional study with 74 aged was conducted, excluding the aged who had low educational level. Data collection consisted of a questionnaire application to evaluate the epidemiologic characteristics and the facts concerning health and the Mental State Small-Exam. Results: Among the evaluated elderly, we found a prevalence in a cognitive decline of $36.5 \%$. The score average in the MEEM was 19.49 points and the average in the aged was 68.5 years old. Discussion: The results show that the small level in frequency to school has a negative influence in the metal state in the elderly. Conclusion: It is high the number of elderly with cognitive decline, and the result shows that the MEEM influences educational level, what makes it difficult to interpret the results in elderly with low educational level.
Key words: Elderly. Evaluation. Cognitive decline. Mental state small exam. Dementia.

\section{INTRODUÇÃO}

A demência é uma das mais importantes causas de morbi-mortalidade entre os idosos, e é caracterizada como uma síndrome crônica cujas características principais são representadas pelo declínio da memória, declínio intelectual e de outras funções como: linguagem, praxia, capacidade de reconhecer e identificar objetos, abstração, organização, capacidade de planejamento e sequenciamento, mudanças no comportamento ou na personalidade, além do prejuízo no desenvolvimento psicossocial. O grau de incapacidade aumenta com o avanço do declínio cognitivo. ${ }^{1}$

Atualmente, a demência é o problema de saúde mental que mais cresce em importância e número, com estimativas apontando que sua incidência anual cresce sensivelmente com o envelhecimento, de $0,6 \%$, na faixa dos 65-69 anos, para $8,4 \%$ naqueles com mais de 85 anos; sua prevalência passa de $5 \%$ nos idosos com mais de 60 anos para $20 \%$ naqueles com mais de 80 anos. ${ }^{2,3}$

De acordo com Lopes e Botino, a prevalência média de demência na população variou entre 2,2\% na África, 5,5\% na Ásia, 6,4\% na América do Norte, $7,1 \%$ na América do Sul e 9,4\% na Europa. No Brasil, descreveu-se uma taxa de prevalência intermediária de $7,1 \%{ }^{4}$

Estudo realizado no município de Santo Antônio de Pádua, Rio de Janeiro, avaliou 341 idosos pelo
Mini-Exame do Estado Mental e encontrou uma prevalência de declínio cognitivo de $16,61 \% .{ }^{5} \mathrm{Já}$ em outro estudo realizado em Catanduva, São Paulo, em que foram avaliados 1.660 idosos, constatou-se uma prevalência de $7,1 \%{ }^{6}$

As síndromes demenciais são caracterizadas pela presença de declínio cognitivo progressivo e representam um significativo problema de saúde pública, pela longa extensão e complexidade de manifestações funcionais, emocionais e consequências sociais, tanto para a pessoa idosa afetada quanto para seus familiares. ${ }^{7,8,9}$

O comprometimento cognitivo afeta a capacidade funcional do indivíduo no seu dia a dia, implicando perda de independência e autonomia, a qual varia de acordo com o grau de gravidade, com consequente perda da qualidade de vida do idoso. A noção de autonomia, no que tange à interdependência desta com uma memória íntegra, reside na capacidade individual de cuidar de si mesmo, executar tarefas que lhe permitam a adaptação psicosocial e ser responsável pelos próprios atos. ${ }^{10,11}$

Segundo Veras et al., ${ }^{12}$ o impacto da demência (e de desordens a ela relacionadas) sobre a família do indivíduo e a sociedade não deve ser subestimado. Vários são os fatores de risco a ela associado, tais como: a natureza marcadamente dependente da idade, que caracteriza a deficiência cognitiva, a predominância de mulheres (como resultado do fator idade), a acentuada importância 
dos fatores socioeconômicos e associação com a incapacidade de desempenhar as atividades cotidianas.

O estudo específico das capacidades cognitivas da população idosa objetiva avaliar, num primeiro estágio, a prevalência de declínio cognitivo. Diversos instrumentos foram desenvolvidos, nos últimos anos, com o objetivo de auxiliar na investigação de declínio cognitivo em indivíduos de risco, como é o caso dos idosos. O Mini-Exame do Estado Mental (MEEM) é a escala mais utilizada para rastreamento do comprometimento cognitivo. ${ }^{2,15}$

O Mini-Exame do Estado Mental (MEEM) é uma escala de avaliação cognitiva que auxilia a investigação e a monitoração da evolução de declínio cognitivo em indivíduos com risco de demência, como o caso dos idosos. Desde que foi proposto por Folstein et al., ${ }^{13}$ vem sendo amplamente utilizada no mundo. No Brasil, o MEEM foi inicialmente proposto por Bertolucci et al., ${ }^{14}$ os quais observaram que o escore total do MEEM dependia do nível educacional. A associação entre o baixo escore do MEEM e a idade avançada também já foi descrita, mas sua associação com outros fatores ainda não foi investigada em estudos de base populacional. ${ }^{2,15}$

O declínio cognitivo interfere na realização de atividades sociais e ocupacionais, evoluindo para a perda das capacidades funcionais ou da autonomia, de acordo com o estágio da enfermidade. $\mathrm{O}$ diagnóstico de demência tem como base a investigação de declínio cognitivo em populações de risco com instrumentos como o Mini-Exame do Estado Mental. ${ }^{8,15}$ Nesse sentido, faz-se necessário realizar estudos epidemiológicos sobre demência. Conhecendo mais sobre sua etiologia e prevalência, pode-se contribuir para a orientação mais efetiva das políticas públicas de saúde e, assim, melhorar a qualidade de vida no âmbito individual e/ou coletivo.

Com esse propósito, este estudo teve por objetivo analisar, em um grupo de idosos frequentadores do Programa Municipal da
Terceira Idade, em Viçosa, Minas Gerais, a prevalência de declínio cognitivo e sua relação com as enfermidades autorreferidas e fatores relacionados à situação de saúde.

\section{METODOLOGIA}

\section{Desenho do Estudo}

Trata-se de um estudo epidemiológico em que se utilizou o método analítico do tipo transversal para investigar a prevalência de declínio cognitivo e sua relação com as características epidemiológicas e fatores relacionados à situação de saúde dos idosos, participantes do Programa Municipal da Terceira Idade do município de Viçosa-MG. Este programa foi instituído em 1997, sendo resultado de uma parceria entre o Núcleo de Saúde Pública (NUSP) do Departamento de Nutrição e Saúde (DNS), Universidade Federal de Viçosa (UFV), com a Prefeitura Municipal de Viçosa (PMV), tendo como objetivo principal promover, por meio de um processo educativo-preventivo, a melhoria da qualidade de vida dos idosos. Segundo o último cadastro (agosto de 2005), o PMTI atende 1.025 idosos, os quais participam de atividades físicas e de recreação, atendimento médico, odontológico e nutricional.

\section{Seleção da Amostra}

Os idosos foram selecionados para o estudo de forma aleatória simples, a partir da lista de cadastrados do PMTI. Excluíram-se do sorteio os idosos que apresentaram deficiências físicas e mentais, como: limitação na coordenação motora fina, surdez, dificuldade visual e/ou qualquer tipo de demências já diagnosticada, uma vez que estas poderiam interferir no resultado do teste MEEM.

Tendo em vista que a variável escolaridade também influencia no escore de declínio cognitivo, só foram incluídos no estudo os idosos com pelo menos um ano de estudo formal. ${ }^{2}$

A amostra foi calculada considerando uma prevalência de declínio cognitivo de no mínimo 
$5 \%$ da população de idosos. ${ }^{13}$ Definiu-se um poder de estudo de $90 \%$ e um erro de $5 \%{ }^{17}$. Deste modo, foram avaliados 74 idosos.

\section{Coleta e Análise dos Dados}

Foi criado um questionário específico para análise dos aspectos epidemiológicos e dos fatores relacionados à situação de saúde. Para investigar a presença de declínio cognitivo, utilizou-se o teste do Mini-Exame do Estado Mental (MEEM).

Os dados foram obtidos a partir de entrevistas individuais na sede do programa, realizadas por uma única pesquisadora. No questionário foram coletadas as seguintes variáveis:

a. Características gerais dos idosos: sexo, cor, faixa etária, grau de instrução e estado civil.

b. Fatores relacionados à situação de saúde: se faz atividade física regularmente, o tipo e a frequência, sendo considerada como adequada, uma prática com duração maior ou igual a 30 minutos e com uma frequência mínima de três vezes por semana. Foram considerados tabagistas os indivíduos que faziam uso de qualquer tipo de cigarro, independentemente da frequência e da quantidade, sendo que os mesmos critérios foram usados para o etilismo. Questionouse também sobre a ocorrência de queda nos últimos 12 meses.

c. Enfermidades autorreferidas e autopercepção do estado de saúde.

Para identificar o declínio cognitivo, foi utilizado o teste psicométrico do Mini-Exame do Estado Mental (MEEM), versão em português, validada por Bertolucci et al.. ${ }^{14} \mathrm{O}$ MEEM é um teste de rastreio e triagem mais utilizado no mundo, pois é simples, de aplicação rápida e autoexplicativo. É composto por diversas questões tipicamente agrupadas em sete categorias, cada uma delas formulada com o objetivo de avaliar funções cognitivas específicas: orientação, retenção ou registro de dados, atenção e cálculo, memória e linguagem. O escore do MEEM pode variar de um mínimo de 0 até o total máximo de 30 pontos. Tombaugh \& McIntyre ${ }^{18}$ observaram que a escala tem boa consistência interna e confiabilidade testereteste $(0,8 \%$ a $0,95 \%)$ e que o ponto de corte 23 / 24 tem de boa a excelente sensibilidade e especificidade para o diagnóstico de demência. ${ }^{2}$

Para a interpretação dos resultados, considerou-se a existência de associação entre um determinado fator de estudo (enfermidades autorreferidas e fatores relacionados à situação de saúde) e a presença de declínio cognitivo.

Os dados foram digitados em um banco de dados no programa Epi-Info versão 6.02. Foram realizadas análise descritiva e análise bivariada, bem como estimados os valores de odds ratio brutas com intervalos de confiança de $95 \%$.

O protocolo de pesquisa foi aprovado pelo Comitê de Ética em Pesquisa com Seres Humanos, da Universidade Federal de Viçosa, Minas Gerais. Todos os indivíduos que aceitaram participar do estudo assinaram um termo de consentimento livre esclarecido.

\section{RESULTADOS}

Como descrito na tabela 1 , dos 74 idosos entrevistados, 27 obtiveram escore abaixo de 23 pontos, correspondendo a uma prevalência 36,5\% de declínio cognitivo entre os idosos da amostra. A média de pontuação no MEEM da amostra total foi de 19,48 pontos (menor pontuação $=$ 13 ; maior pontuação $=28$ ).

A idade do grupo variou de 60 a 85 anos. Constatou-se uma predominância de idosos com idade entre 60 e 69 anos (65\%), seguidos dos que têm entre 70 e 79 anos (32\%), e dos que têm mais de 80 anos (3\%). Quando se relaciona a faixa etária com o declínio cognitivo, verifica-se também que a maioria dos idosos com declínio cognitivo tem idade entre 60 e 69 anos $(24,32 \%)$.

Os idosos estudados apresentam um baixo nível de escolaridade, com uma proporção de $91 \%$ de idosos com menos de quatro anos de estudo. Quanto à situação conjugal, constatou-se que a 
maioria dos idosos é casada (60\%) e que, em relação ao arranjo familiar no domicílio, $28 \%$ moram com cônjuge; $46 \%$ moram com o cônjuge ou não e filhos; $12 \%$ moram com o cônjuge ou não, filhos e netos; e 14\% moram sozinhos.

$\mathrm{Na}$ análise dos fatores associados ao declínio cognitivo, chama atenção a associação entre a escolaridade e a presença de declínio cognitivo. A razão de chances (odds ratio) mostra que os idosos com um ano de estudo ou menos tem 3,83 vezes mais chances de ter declínio cognitivo do que os que tem de um a quatro anos de estudos [OR $=3,83$; IC $(1,18 ; 12,88)]$. A associação das demais variáveis sociodemográficas com o declínio cognitivo não apresentou significância estatística.

Tabela 1 - Distribuição dos idosos com declínio cognitivo e sem declínio cognitivo segundo fatores sociodemográficos. Viçosa, MG, 2005.

\begin{tabular}{|c|c|c|c|c|c|}
\hline Variáveis & Categorias & $\begin{array}{c}\text { Total } \\
\%\end{array}$ & $\begin{array}{l}\text { Com Declínio } \\
\text { Cognitivo } \\
\%\end{array}$ & $\begin{array}{c}\text { Sem Declínio } \\
\text { Cognitivo } \\
\%\end{array}$ & $\bar{O}$ R/IC 95\% \\
\hline & $60-69$ anos & 65 & 24,32 & 40,5 & 1,0 \\
\hline \multirow{2}{*}{ Faixa etária } & $70-79$ anos & 32 & 10,81 & 21,6 & $1,20(0,38-3,82)$ \\
\hline & 80 ou mais & 3 & 1,75 & 1,35 & $0,60(0,02-23,69)$ \\
\hline \multirow[t]{3}{*}{ Sexo } & Masculino & 26 & 4,1 & 21,6 & $0,24(0,05-1,04)$ \\
\hline & Feminino & 74 & 32,4 & 41,9 & \\
\hline & Branca & 14 & 6,75 & 6,75 & 1,0 \\
\hline \multirow[t]{2}{*}{ Cor } & Preta & 19 & 8,1 & 10,8 & $1,33(0,19-9,33)$ \\
\hline & Parda & 67 & 21,62 & 45,98 & $2,13(0,45-10,23)$ \\
\hline Escolaridade & 1 ano & 50 & 25,67 & 22,9 & 1,0 \\
\hline completa & $1-4$ anos & 41 & 9,45 & 32,4 & $3,83(1,18-12,88)$ \\
\hline (em Anos) & 4 ou mais & 9 & 1,75 & 8,1 & $6,71(0,66-63,53)$ \\
\hline Estado Civil & $\begin{array}{l}\text { Solteiro/Viúvo/Se } \\
\text { parado } \\
\text { Casado }\end{array}$ & $\begin{array}{l}40 \\
60\end{array}$ & $\begin{array}{l}17,59 \\
18,9\end{array}$ & $\begin{array}{l}22,97 \\
40,54\end{array}$ & $0,61(0,21-1,78)$ \\
\hline
\end{tabular}

Quanto à prática de atividades físicas, verificase na tabela 2 , que $51 \%$ dos idosos fazem alguma atividade, sendo que $79 \%$ com uma regularidade de três vezes ou mais por semana. A modalidade mais praticada foi a caminhada (28\%), realizada na maioria das vezes sem orientação profissional (53\%). A respeito do tabagismo, a maioria dos idosos respondeu que não fuma atualmente (85\%), e ainda, $64 \%$ responderam que nunca fumaram. Quanto ao etilismo, $89 \%$ não bebe atualmente, e $81 \%$ também não beberam durante a vida.

Entre aqueles com declínio cognitivo, o número de fumantes e ex-fumantes também foi de $6,75 \%$ e $12,16 \%$, respectivamente e, com relação ao consumo de álcool, o estudo mostrou que $4 \%$ bebem ou já beberam.

Os resultados deste estudo mostram que $24 \%$ dos idosos já sofreram algum tipo de queda no último ano, sendo que, dos que sofreram queda, 10,81\% apresentaram declínio cognitivo e 13,5\%, não.

Quanto à relação entre o declínio cognitivo e os fatores associados à situação de saúde, observou-se que os idosos que fazem menos atividades físicas, considerados tabagistas, etilistas e que já tiveram quedas, têm mais chances de apresentar declínio cognitivo, embora os resultados não tenham sido estatisticamente significantes. 
Tabela 2 - Distribuição dos idosos com declínio cognitivo e sem declínio cognitivo segundo fatores relacionados à situação de saúde. Viçosa, MG, 2009.

\begin{tabular}{|c|c|c|c|c|c|}
\hline Variáveis & Categorias & $\begin{array}{c}\text { Total } \\
\%\end{array}$ & $\begin{array}{c}\text { Com Declínio } \\
\text { Cognitivo } \\
\%\end{array}$ & $\begin{array}{c}\text { Sem Declínio } \\
\text { Cognitivo } \\
\%\end{array}$ & OR /IC 95\% \\
\hline \multirow{2}{*}{$\begin{array}{l}\text { Faz atividade } \\
\text { física } \\
\text { regularmente }\end{array}$} & $\overline{\operatorname{Sim}}$ & 51 & 18,9 & 32,43 & \multirow[t]{2}{*}{$0,99(0,35-2,83)$} \\
\hline & Não & 49 & 17,59 & 31,08 & \\
\hline \multirow{4}{*}{$\begin{array}{l}\text { Tipo de } \\
\text { atividade } \\
\text { física }\end{array}$} & Caminhada & 28 & 12,16 & 16,21 & \multirow[t]{4}{*}{$1,95(0,42-9,33)$} \\
\hline & Ginástica & 24 & 6,75 & 17,56 & \\
\hline & Hidroginástica & & & & \\
\hline & $\begin{array}{l}\text { 1-2 Vezes } \\
\text { por Semana }\end{array}$ & 16 & 2,7 & 5,4 & \\
\hline \multirow{2}{*}{$\begin{array}{l}\text { Frequência da } \\
\text { atividade física }\end{array}$} & $\begin{array}{c}3 \text { ou Mais Vezes } \\
\text { por Semana }\end{array}$ & 79 & 12,16 & 28,37 & \multirow[t]{2}{*}{$1,7(0,12-9,87)$} \\
\hline & Não Sabem & 5 & 2,7 & 0 & \\
\hline \multirow{2}{*}{$\begin{array}{l}\text { Atividade física } \\
\text { com orientação } \\
\text { profissional }\end{array}$} & Sim & 47 & 6,75 & 17,56 & \multirow[t]{2}{*}{$0,51(0,11-2,37)$} \\
\hline & Não & 53 & 10,81 & 16,21 & \\
\hline \multirow{2}{*}{ Fuma atualmente } & Sim & 15 & 6,75 & 8,1 & \multirow[t]{2}{*}{$1,55(0,36-6,68)$} \\
\hline & Não & 85 & 29,7 & 55,45 & \\
\hline \multirow{2}{*}{ Já fumou } & Sim & 32 & 12,16 & 20,27 & \multirow[t]{2}{*}{$1,07(0,35-3,27)$} \\
\hline & Não & 68 & 24,33 & 43,24 & \\
\hline \multirow{2}{*}{ Bebe atualmente } & Sim & 11 & 4 & 6,75 & \multirow[t]{2}{*}{$1,05(0,18-5,72)$} \\
\hline & Não & 89 & 32,55 & 56,7 & \\
\hline \multirow{2}{*}{ Já Bebeu } & Sim & 19 & 4 & 14,86 & \multirow{2}{*}{$\begin{array}{c}0,41 \\
(0,08-1,84)\end{array}$} \\
\hline & Não & 89 & 32,54 & 48,6 & \\
\hline \multirow{2}{*}{$\begin{array}{l}\text { Sofreu alguma queda } \\
\text { no último ano }\end{array}$} & Sim & 24 & 10,81 & 13,5 & \multirow{2}{*}{$\begin{array}{l}1,56 \\
(046-5,23)\end{array}$} \\
\hline & Não & 76 & 25,69 & 50 & \\
\hline
\end{tabular}

$\mathrm{Na}$ tabela 3, observa-se a distribuição das doenças autorreferidas pelos idosos e classificadas segundo o CID-10. A doença mais referida foi a hipertensão arterial (70\%), seguida por síndrome amnésica orgânica, hipercolesterolemia, problemas osteoarticulares e tonteira e instabilidade.

No que se refere à autopercepção da saúde, observou-se que a maioria dos idosos percebeu sua saúde como boa (72\%) e sua saúde com relação aos outros como melhor ou igual $(23 \%, 61 \%)$, sendo que os idosos sem declínio cognitivo relataram estes fatores com maior frequência.

Verificou-se nas análises que os idosos com declínio cognitivo tiveram mais chances de apresentar hipercolesterolemia, tonteira e instabilidade, depressão e gastrite, embora os resultados não tenham sido estatisticamente significantes. Uma associação significante foi 
encontrada apenas entre a presença de declínio cognitivo e problema osteoarticular. Assim, idosos com declínio cognitivo diminuem em $85 \%$ a chance de problema osteoarticular.
Neste estudo, obteve-se uma associação inversa entre o declínio cognitivo e uma boa autopercepção da saúde; assim, quem tem declínio cognitivo tem a chance $59 \%$ menor de perceber sua saúde como ruim.

Tabela 3 - Distribuição dos idosos com declínio cognitivo e sem declínio cognitivo segundo enfermidades auto-referidas e avaliação subjetiva de saúde. Viçosa, MG, 2005.

\begin{tabular}{|c|c|c|c|c|c|}
\hline Variáveis & Categorias & $\begin{array}{l}\text { Total } \\
\%\end{array}$ & $\begin{array}{c}\text { Com Declínio } \\
\text { Cognitivo } \\
\% \\
\end{array}$ & $\begin{array}{c}\text { Sem Declínio } \\
\text { Cognitivo } \\
\% \\
\end{array}$ & OR/IC 95\% \\
\hline \multirow[t]{2}{*}{ Hipertensão Arterial } & Sim & 70 & 25,67 & 44,6 & $1,01(0,32-3,22)$ \\
\hline & Não & 30 & 10,81 & 18,92 & \\
\hline \multirow{2}{*}{$\begin{array}{l}\text { Síndrome amnésica } \\
\text { orgânica }\end{array}$} & $\operatorname{Sim}$ & 42 & 14,86 & 27,04 & $0,93(0,32-2,7)$ \\
\hline & Não & 58 & 21,62 & 36,48 & \\
\hline \multirow[t]{2}{*}{ Hipercolesterolemia } & Sim & 43 & 17,59 & 25,67 & $1,37(0,47-3,96)$ \\
\hline & Não & 57 & 18,91 & 37,80 & \\
\hline \multirow[t]{2}{*}{ Problema osteoarticular } & Sim & 39 & 5,4 & 33,78 & $0,15(0,04-0,57)$ \\
\hline & Não & 61 & 31,08 & 29,72 & \\
\hline \multirow[t]{2}{*}{ Tonteira e instabilidade } & Sim & 19 & 8,1 & 10,81 & $1,39(0,37-5,25)$ \\
\hline & Não & 81 & 28,39 & 52,7 & \\
\hline \multirow[t]{2}{*}{ Diabetes } & Sim & 18 & 4,05 & 13,51 & $0,46(0,09-2,11)$ \\
\hline & Não & 82 & 32,44 & 50 & \\
\hline \multirow{2}{*}{$\begin{array}{l}\text { Distúrbio } \\
\text { comportamentais }\end{array}$} & $\operatorname{Sim}$ & 11 & 2,7 & 8,1 & $0,55(0,07-3,38)$ \\
\hline & Não & 89 & 33,8 & 55,4 & \\
\hline \multirow[t]{2}{*}{ Depressão } & Sim & 6 & 2,7 & 4,05 & $1,17(0,13-9,52)$ \\
\hline & Não & 94 & 33,8 & 59,45 & \\
\hline \multirow[t]{2}{*}{ Gastrite } & Sim & 4 & 1,35 & 1,35 & $1,77(0,0-68,16)$ \\
\hline & Não & 96 & 35,14 & 62,16 & \\
\hline \multirow[t]{2}{*}{ Trans tornos tireoidianos } & Sim & 5 & 4,05 & 1,35 & $5,75(0,49-151,87)$ \\
\hline & Não & 95 & 32,43 & 62,17 & \\
\hline \multirow[t]{3}{*}{ Como percebe sua saúde } & Ótimo & 12 & 1,35 & 10,81 & $5,68(0,63-129,8)$ \\
\hline & Bom & 72 & 29,72 & 41,9 & 1,00 \\
\hline & Ruim & 16 & 5,4 & 10,81 & $0,41(0,13-1,26)$ \\
\hline \multirow{3}{*}{$\begin{array}{l}\text { Sua Saúde em Relação } \\
\text { aos Outros }\end{array}$} & Melhor & 23 & 6,75 & 16,21 & $1,60(0,42-6,33)$ \\
\hline & Igual & 61 & 24,32 & 36,51 & 1,00 \\
\hline & Pior & 16 & 5,4 & 10,81 & $1,33(0,30-6,28)$ \\
\hline
\end{tabular}




\section{DISCUSSÃO}

Encontrou-se uma alta prevalência de declínio cognitivo entre os idosos estudados. No entanto, é importante considerar que o ponto de corte utilizado ( 23 pontos) não foi ajustado pela escolaridade e, neste caso, o mesmo seria alto para idosos com baixa escolaridade. No entanto, como é desejável neste estudo, aumentaria a sensibilidade do teste para a detecção de um número maior de casos verdadeiros, ao mesmo tempo em que diminuiu a possibilidade de idosos com um grau "leve" de declínio cognitivo não serem diagnosticados.

Segundo as Recomendações do Departamento Científico de Neurologia Cognitiva e do Envelhecimento da Academia Brasileira de Neurologia"(2005), ${ }^{19}$ na utilização do MEEM como instrumento de rastreio para demência, é necessário empregar pontos de corte mais elevados para aumentar a sensibilidade do teste. ${ }^{20}$ Nitrini et al. ${ }^{19}$ sugerem "o uso de outro(s) instrumento(s) aposteriori para a confirmação de perda cognitiva em indivíduos com escores abaixo de: analfabetos (20); 1- 4 anos de estudo (25), 4- 8 anos (26,5), 9 -11 anos (28)”.

Laks et al., ${ }^{21}$ em 2007, realizaram um estudo para avaliar normas do MEEM de acordo com a idade e escolaridade de idosos vivendo em comunidade. O MEEM total encontrado foi 21,97 (desvio-padrão $=4,48$ ). Nos indivíduos mais jovens, o MEEM entre os idosos com 1-8 anos de escolaridade foi 23,90 (desvio-padrão $=3,87$ ) e a distribuição dos quartis foi 15, 22, 24, 27. Nos mais idosos, a pontuação dos sujeitos com 1-8 anos foi 23,81 (desvio-padrão $=4,01$ ) e quartis de 15, 22, 24, 27.

Os estudos sobre prevalência da demência enfrentam grandes obstáculos, tais como ausência de teste diagnóstico que possa ser aceito como "padrão-ouro" e dificuldade na utilização de instrumentos de rastreio validados em outros países que podem ter acurácia muito diferente, dependendo de fatores culturais e sociais, necessitando de adaptação de itens ou de modificação dos escores de corte. ${ }^{3}$

Os idosos estudados têm em média 68,5 anos e são, na maioria, do sexo feminino, de cor parda, casados e de baixa escolaridade. Os resultados relativos ao gênero confirmam a tendência encontrada na literatura nos estudos relacionados com os idosos segundo os quais, no Brasil, o número absoluto de mulheres idosas tem sido superior quando confrontado com o de homens acima de 65 anos.

No estudo de Lebrão e Laurenti ${ }^{22}$ sobre saúde, bem-estar e envelhecimento, realizado em São Paulo, com 2.143 idosos, encontrou-se a porcentagem de $58,6 \%$ de mulheres, valor bastante próximo ao encontrado no censo de 2000 , que foi de $59,5 \% .{ }^{23}$ A predominância da população feminina entre os idosos tem causado repercussões nas demandas por políticas públicas de saúde. Isso porque as mulheres estão mais sujeitas a deficiências físicas e mentais, há uma elevada proporção de mulheres morando sozinhas em decorrência da viuvez (14\%, em 1998), elas têm menor nível de escolaridade, menor experiência de trabalho no mercado formal e menor acesso à aposentadoria, o que requer maior assistência tanto do Estado quanto da família. ${ }^{22}$ No que se refere à baixa escolaridade, a proporção $\mathrm{da}$ população de idosos alfabetizada é baixa. Segundo o censo de $2000,41,1 \%$ da população acima de 60 anos eram analfabetos. ${ }^{23}$

Considerando que o PMTI incentiva a prática de atividades físicas e oferece aulas de ginástica e hidroginástica, observa-se que ainda há um número grande de idosos sedentários e que praticam atividades físicas sem orientação profissional. Desta forma, deve-se investir de maneira efetiva em programas de suporte aos idosos, destacando-se a importância das atividades físicas, pois estas, mesmo se iniciadas após os 65 anos, trazem benefícios, tais como: maior longevidade; melhora da capacidade fisiológica de portadores de doenças crônicas; redução do número de medicamentos prescritos; prevenção do declínio cognitivo; manutenção do status funcional mais elevado; redução da frequência de quedas e incidência de fraturas; benefícios psicológicos, como a melhora da autoestima. ${ }^{24}$

A respeito do tabagismo e etilismo, a maioria dos idosos respondeu que não fuma e nem bebe atualmente, e ainda, a grande maioria nunca bebeu 
e nem fumou durante a vida. Entre aqueles com declínio cognitivo, esses números também foram pequenos. Isso é especialmente importante quando se considera que, no idoso, o hábito de fumar acelera a perda da densidade óssea, da força muscular e da função respiratória, além de estar negativamente relacionada a outros fatores causadores da perda da capacidade funcional e interferir no efeito de alguns medicamentos. $\mathrm{O}$ consumo de álcool também pode aumentar a predisposição do idoso a certas enfermidades como, por exemplo, desnutrição, enfermidades hepáticas, gástricas e pancreáticas, bem como interferir no efeito de medicamentos e aumentar o risco de quedas. ${ }^{25}$

Pelos resultados obtidos neste estudo, destacase que uma parcela significativa de idosos já sofreu algum tipo de queda no último ano. Tais informações podem ser importantes no planejamento de programas de prevenção de quedas para essa população. Estudos realizados em comunidades americanas mostram que $30 \%$ das pessoas com idade superior a 65 anos caem pelo menos uma vez ao ano, sendo que $40 \%$ delas têm idade superior a 80 anos. As quedas em idosos têm como consequências, além de possíveis fraturas e risco de morte, o comprometimento progressivo em sua capacidade funcional ao longo do tempo, na medida em que, pelo medo de cair, os idosos podem encontrar-se em uma situação de baixa autoconfiança para realizar atividades do dia a dia. ${ }^{26}$

Um dado relevante é o alto percentual de idosos com hipertensão arterial. Dentre as outras enfermidades mais mencionadas estão a síndrome amnésica orgânica, a hipercolesterolemia e os problemas osteoarticualres. Segundo Pacheco e Santos, ${ }^{27}$ as principais doenças que acometerão os idosos, além das mais conhecidas doenças crônicas não-transmissíveis (hipertensão, diabetes e neoplasias), serão: demência - principalmente do tipo doença de Alzheimer; depressão e osteoporose - as quais requerem cuidados especializados, dos quais os países em desenvolvimento não se encontram preparados para atender as necessidades biopsicossociais dessa população.
Estudos mostram que as doenças cardiovasculares foram a primeira causa de morte entre os idosos brasileiros, aumentando de forma acentuada com a idade, seguido das neoplasias, doenças do aparelho respiratório e doenças endócrinas. A mortalidade por essas causas pode ser devida, pelo menos em parte, à presença de fatores de riscos modificáveis como o fumo, inatividade física, obesidade, dislipidemia e controle inadequado da hipertensão e do diabete..$^{28-31}$

Quanto à autopercepção do estado de saúde, estudos recentes têm mostrado que os indivíduos que relatam condições de saúde ruim correm risco de mortalidade consistentemente mais altos do que aqueles que reportam melhor estado de saúde. ${ }^{32}$

Neste estudo, os resultados referentes à autopercepção do estado de saúde foram positivos, pois mostraram que a maioria dos idosos percebeu sua própria saúde, inclusive com relação à saúde dos outros, como boa, sendo que os idosos sem declínio cognitivo relataram estes fatores com maior frequência.

A autoavaliação da saúde é um dos indicadores mais usados em pesquisas gerontológicas, porque prediz de forma consistente a mortalidade e o declínio funcional. Além disso, a autoavaliação da saúde reflete uma percepção integrada do indivíduo, que inclui as dimensões biológicas, psicossocial e social. Um estudo epidemiológico com 1.516 idosos, conduzido em Bambuí, Minas Gerais, sobre os fatores associados à autoavaliação da saúde, mostrou que $24,7 \%$ consideram sua saúde como boa ou muito boa, $49,2 \%$ consideram razoável, e $26,1 \%$ ruim ou muito ruim. ${ }^{32}$ Já no estudo de Lebrão e Laurenti, ${ }^{22}$ anteriormente mencionado, as autoavaliações de saúde mostraram que $53,8 \%$ dos idosos consideram sua saúde regular ou má e 46,2\% consideram excelente, muito boa ou boa.

Quando se analisou a relação do declínio cognitivo com as variáveis faixa etária, sexo, cor, escolaridade e estado civil, descritos na tabela 4 a razão de chances (odds ratio) entre a variável 
escolaridade e o declínio cognitivo indica que os idosos com um ano de estudo ou menos têm 3,83 vezes mais chances de ter declínio cognitivo do que os que têm de uma a quatro anos de estudos.

Tabela 4 - Razão de chances entre as variáveis econômicas sócio-demográficas com a presença ou não de declínio cognitivo. Viçosa, MG, 2005.

\begin{tabular}{lll}
\hline \multicolumn{1}{c}{ Variáveis } & Categorias & OR (IC 95\%) \\
\hline \multirow{2}{*}{ Faixa Etária } & $* 60-69$ anos & 1,0 \\
& $70-79$ anos & $1,20(0,38-3,82)$ \\
Sexo & 80 ou mais & $0,60(0,02-23,69)$ \\
& Masculino & $0,24(0,05-1,04)$ \\
& Feminino & \\
Cor & *Branca & 1,0 \\
& Preta & $1,33(0,19-9,33)$ \\
Escolaridade Completa (em Anos) & Parda & $2,13(0,45-10,23)$ \\
& $* 1$ ano & 1,0 \\
& $1-4$ anos & $3,83(1,18-12,88)$ \\
& 4 ou mais & $6,71(0,66-163,53)$ \\
Estado Civil & Solteiro/Viúvo/ & $0,61(0,21-1,78)$ \\
& Separado & \\
& Casado & \\
\hline
\end{tabular}

*-Valor de referência.

Embora este estudo tenha evidenciado somente a influência da escolaridade com a presença de declínio cognitivo, no Brasil e em países em desenvolvimento, essa associação tem sido encontrada em estudos populacionais sobre demência utilizando como instrumento de avaliação o MEEM. No estudo realizado em Santo Antônio de Pádua, Rio de Janeiro, anteriormente mencionado, foram avaliados 341 idosos acima de 65 anos pelo MEEM. Seu objetivo era definir a pontuação global do MEEM de idosos da comunidade por faixa etária e escolaridade. Nesse estudo, constatou-se que, nos idosos com idade entre 65-84 anos, alfabetizados ou não, a escolaridade e a idade influenciam de modo significativo nos resultados do MEEM. ${ }^{3}$

Já em estudo realizado por Almeida, ${ }^{2}$ com 211 idosos atendidos em um ambulatório de saúde mental da Santa Casa de São Paulo, buscou-se investigar o melhor ponto de corte do MEEM para o diagnóstico de demência e o impacto da escolaridade e da idade sobre o escore total. Os resultados desse estudo confirmaram que a idade e a escolaridade se associam de forma significativa com o desempenho dos idosos pelo MEEM.

A razão de chances entre os fatores relacionados à situação de saúde e o declínio cognitivo não mostrou significância estatística. No entanto, em relação à ocorrência de quedas, Carvalho \& Coutinho ${ }^{33}$ relatam que várias são as características da demência que podem estar relacionadas ao aumento de quedas nos idosos como: comprometimento da atenção e da memória, apraxia, agnosia, desorientação espacial, deterioração das funções executivas dentre outras. Esses autores realizaram um estudo sobre a demência como fator de risco para fraturas graves em idosos, confirmando que idosos com quadro demencial apresentam maior risco de caírem e serem hospitalizados por fraturas do que idosos sem demência. Acidentaram-se dentro de casa 78\% dos idosos com demência, contra 55\% daqueles 
sem a doença. A odds-ratio (OR) não ajustada mostrou associação positiva [OR =2,0; IC 95\% $(1,23-3,2)]$. Após o ajuste por fatores de confusão, houve uma pequena redução dessa associação $[\mathrm{OR}=1,82,1,03-3,23]$.

Outros autores encontraram que as chances de queda aumentam entre mulheres, idosos viúvos, solteiros ou desquitados, idosos com história prévia de fraturas, com grau de comprometimento nas atividades de vida diária e entre os que não referem a leitura como atividade de lazer, porque a leitura envolve algumas habilidades cognitivas específicas como atenção e concentração. Além disso, eles mostraram a importância da função cognitiva, especialmente a atenção, como um importante fator protetor para queda. ${ }^{34}$

Ao se analisar a associação entre enfermidades autoreferidas e declínio cognitivo observou-se que os idosos com declínio cognitivo relataram menos problemas osteoarticulares do que os idosos sem declínio cognitivo, com associação estatisticamente significante. Tal achado pode ser explicado pela maior frequência de atividade física sem orientação profissional relatada por este grupo. Entretanto, em outros estudos, há relatos de que os indivíduos com declínio cognitivo estariam mais sujeitos a terem problema osteoarticular. ${ }^{33}$

\section{CONCLUSÃO}

A população estudada é predominantemente do sexo feminino, com idade entre 60-69 anos, casados e com baixo nível de escolaridade. Os fatores relacionados à saúde mostram que o sedentarismo ainda é alto entre os idosos do programa, a grande maioria não tem ou teve hábitos indesejáveis como o fumo e o álcool.
Dentre as enfermidades autorreferidas, destacase a hipertensão, seguida em menor número, mas também significativo, pela hipercolesterolemia e a síndrome amnésica orgânica.

Constatou-se uma alta prevalência de idosos com declínio cognitivo. Tal resultado revela que o teste MEEM é influenciado pela a variável escolaridade e, como mostrado em outros estudos, a utilização de testes cognitivos combinados com relatos dos informantes pode melhorar o rastreio de demência leve a moderada em idosos com baixo nível educacional. ${ }^{4,35} \mathrm{Cabe}$ lembrar que, em nosso país, o ensino fundamental é bastante heterogêneo, com características regionais próprias, o que faz com que se tenha heterogeneidade no perfil de respostas. Isso é de particular importância em estudos populacionais de declínio cognitivo, nos quais diversos estudos procuraram determinar o ponto de corte ajustado à escolaridade e estabelecer condutas padronizadas na aplicação do MEEM. O consenso da Academia Brasileira de Neurologia sugere uniformizar as avaliações, para a comparação entre os estudos. ${ }^{19}$

Os resultados indicam a necessidade de reforçar a importância do diagnóstico precoce do declínio cognitivo, o que permite o tratamento mais eficaz e possibilita desenvolver ações efetivas que promovam a melhoria na qualidade de vida dos idosos.

Contudo, há necessidade de um maior número de pesquisas que avaliem a presença de declínio cognitivo e seus impactos na qualidade de vida dos idosos. Como limitação deste estudo, ressaltase o número de idosos avaliados; neste sentido, novos estudos devem ser realizados a fim de se avaliar a relação da variável escolaridade com outros fatores. 


\section{REFERENNCIAS}

1. Macedo MBM, Ramos LR. Validade da versão em português da Clinical Dementia Rating. Rev Saúde Pública 2005; 39 (6): 912-17.

2. Almeida OP. Mini exame do estado mental e o diagnóstico de demência no Brasil. Arq NeuroPsiquiatr 1998; 56(3-b): 605-12.

3. Nitrini R. Epidemiologia da doença de Alzheimer no Brasil. Rev Psiquiatr Clín. 1999; $26(5): 1-10$.

4. Lopes MA, Botino CMC. Prevalência de Demência em diversas regiões do mundo: análise dos estudos epidemiológicos de 1994 a 2000. Arq Neuro-Psiquiatr 2002; 60(1): 61-69.

5. Botino CMC, Zevallos-Bustamante SE, Lopes MA. Combined Instruments for the Screening of Dementia in Older People with Low Education. Arq Neuro-Psiquiatr 2009; 67(2-A): 185-190.

6. Herrera Junior E; Caramelli P, Nitrini R. Estudo epidemiológico populacional de demência na cidade de Catanduva. Rev Psiquiatr Clín 1998; 25 (2): 70-73.

7. Laks J. et al. O mini exame do estado mental em idosos de uma comunidade. Dados parciais de Santo Antônio de Pádua. Arq Neuro-Psiquiatr 2003; 61 (3-b): 782-785.

8. Neto JG, Tamelini MG, Forlenza OV. Diagnóstico diferencial das demências. Rev Psiquiatr Clín 2005; 32 (3):119-130.

9. Pelzer MT. A enfermagem e o idosos portador de demência tipo Alzheimer: desafios do cuidar no novo milênio. Est Interdiscipl Envelhec 2002; 4: 97-111.

10. Abreu ID, Forlenza OV, Barros L.H. Demência de Alzheimer : correlação entre memória e autonomia. Rev Psiquiatr Clín 2005; 32 (3): 131-36.

11. Dourado M, et al. Consciência da doença na Demência. Resultados preliminares em pacientes com doença de Alzheimer leve e moderada. Arq Neuro-Psiquiatr 2005; 63 (1):114-118.

12. Veras $\mathrm{R}$,et al. Um envelhecimento digno para o cidadão do futuro. Rio de Janeiro: RelumeDumará; Unati/Universidade Estadual do Rio de Janeiro; 1995.

13. Folstein MS, Folstein SE, McHugh PR. Minimental state: a practical method for grading the cognitive state of patients for the clinician. J Psychiatr Res 1975; 12: 189-198.

14. Bertolucci PHF, Brucki SMD, Campacci SR, Juliano Y. O mini-exame do estado mental em uma população geral: impacto da escolaridade. Arq Neuro-Psiquiatr 1994;52: 1-7.

15. Almeida OP, Crocco EI. Percepção dos déficits cognitivos e alterações do comportamento em pacientes com doença de Alzheimer. Arq Neuro-Psiquiatr 2000; 58: 292-99.

16. Valle EA; Castro-Costa E; Firmo JEA. Estudo de base populacional dos fatores associados ao desempenho no Mini Exame do Estado Mental entre idosos: Projeto Bambuí. Cad Saúde Pública2009; 25 (4):918-926.

17. Lwanga SK, Lemessow S. Sample size determination in health studies: a practical manual. Genebre: World Health Organization; 1982.

18. Tombaugh TN, McIntyre NJ. The mini-mental examination: a comprehensive review. J Am Geriatr Soc 1992; 40: 922-935.

19. Nitrini R, Caramelli P, Bottino AMA, Damasceno BP, Brucki SMD, Anghinah R. Diagnóstico de Doença de Alzheimer no Brasil. Avaliação Cognitiva e Funcional. Arq NeuroPsiquiatr 2005; 63 (3-A): 720-727.

20. Brucki SMD, Nitrini R, Caramelli P, Bertolucci PHF, Okamoto IH. Sugestões para o uso do Mini- Exame do Estado Mental no Brasil. Arq Neuro-Psiquiatr. 2003; 61 (3-B): 777-81 .

21. Laks J., Baptista LMR, Contino ALB, Paula EO, Engelhardt E. Mini-Mental State Examination norms in a community-dwelling sample of elderly with low schooling in Brazil. Cad Saúde Pública 2007; 23 (2).

22. Lebrão ML, Laurenti R. .Saúde, bem-estar e envelhecimento: o estudo SABE no município de São Paulo. Rev Brás Epidemiol 2005; 8(2): 127-41.

23. Instituto Brasileiro de Geografia e Estatística . Dados preliminares do censo de 2000. [acessado em jul 2006]. Disponível em: http:/ www.ibge.gov.br.

24. Chaimowicz F. A saúde dos idosos brasileiros às vésperas do século XXI: problemas, projeções e alternativas. Rev Saúde Pública 1997; 31 (2): 184-200.

25. Hulse G K. Alcohol, drugs and much more in later life. Rev Bras Psiquiatr. 2002; 24 (I): 34-41.

26. Fabrício SCC, Rodrigues AP, Júnior MLC. Causas e consequências de quedas de idosos atendidos em hospital público. Rev Saúde Pública 2004; 38 (1):93-9. 
27. Pacheco RO,Santos SSC. Avaliação global de idosos em unidades de PSF. Textos Envelhecimento 2005; 7: 1-10.

28. Lima e Costa MFF. Diagnóstico da situação de saúde da população idosa brasileira: um estudo da mortalidade e das internações hospitalares públicas. Informe Epidemiológico do SUS 2000; $9(1): 23-41$.

29. Malta DC, Cesário AC, Moura L, Moraes Neto OL, Junior Silva JB. A construção da vigilância e prevenção das doenças crônicas não transmissíveis no contexto do Sistema Único de Saúde. Epidemiologia e Serviços de Saúde 2006;15 (1): 47-65.

30. Venturi I, et al. Os serviços de Saúde e a inclusão da população idosa: uma avaliação das prioridades em saúde. O Mundo da Saúde 2005; 29 (1): 63-69.
31. Camarano AA, Yazaki LM. Envelhecimento da população brasileira: uma contribuição demográfica. Rio de Janeiro: IPEA; 2002.

32. Lima e Costa MFF, Firmo JOA, Uchôa E. A estrutura da auto-avaliação da saúde entre idosos: Projeto Bambuí. Rev Saúde Publica 2004; 38(6): 827-34.

33. Carvalho AM, Coutinho ESF. Demência como fator de risco para fraturas graves em idosos. Rev Saúde Pública 2002;36 (4): 448 - 54.

34. Perracini R R, Ramos LR. Fatores associados a quedas em uma coorte de idosos residentes na comunidade. Rev Saúde Pública 2002; 36: 709-16.

35. Bustamante SEZ, Bottino CMC, Lopes MA, Azevedo D, Hototian SR, Litvoc J, Filho Jacob W. Instrumentos Combinados na Avaliação de Demência em Idosos.Arq Neuro-Psiquiatr 2003; 61 (3-A): 601-06. 
